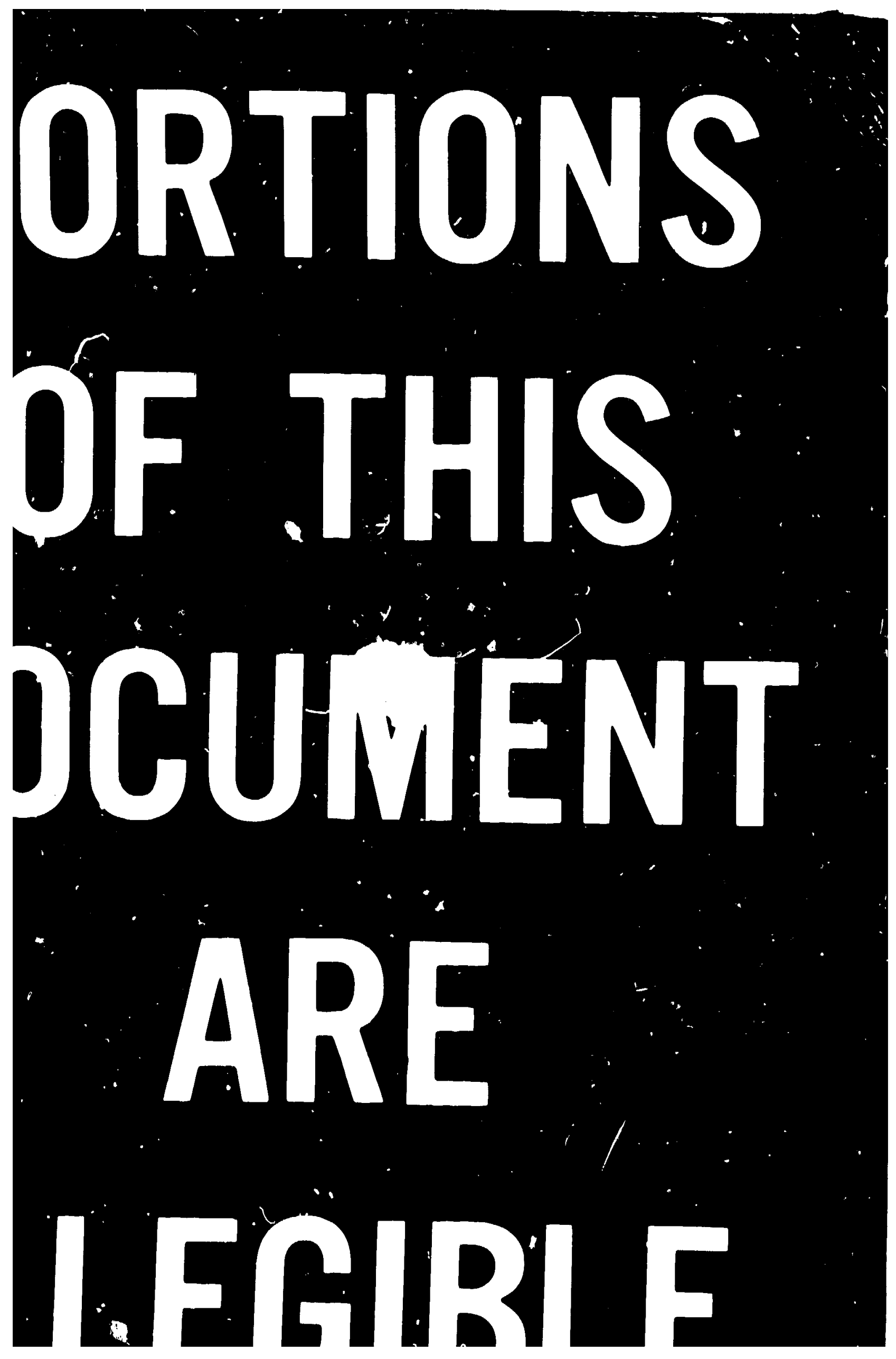


LA-UR-82-340

NOTICE

POATIONS OF THIS REPORT ARE ILLEGIBLE.

It has beent reproduced from the best

available cony to permit the broadest

possibie availability.

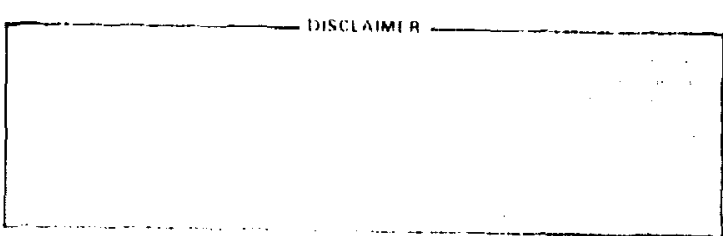

Autmonis) ,

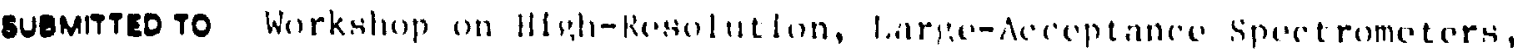

Arsoune Redtomal laboratory, Arsonme, 11., Sipt. 8-11, 1981 
SPECTROAETER DETECTORS

C. L. Morris

\begin{abstract}
Comparisions of different techniques for position measurements in spectrometer focal plane detector systems are made. Capabilities and limits of gas proportionai detectors are discussed.
\end{abstract}

Talk given at the horkshop on High-Resolution, Large-Acceptance spectrometers. Argonne Natlonal Laboratory, September 8-11, 1981. 
I. Int roduction

Although the detectors used to readout positions from a large spectrometer system are a small fraction of the tutal spectrometer system, their performance can have a large impact on the total cost. The better the position resolution the less dispersion that is needed for a given momentum (or energy) resolu:ion, and consequently the smaljer the magnets necessary. Conversely, glven a total size for a spectrometer system, its design should be optimized to makc usa of the position resolution avallable with current state of the art technology.

Most existing medium energy spectrometei gysytems use some version of the gaseous proportional chambers, either aultiwire proportional chambers or drift chambers, to provide position information. First I will try to sumarize the properties and capatilities of gas proportional chambers, and then I will describe the chambers used in some of the existing spectrometer systems, and finally I will present some recent ideas and developoents.

11. Cas Proportlonal Chambers (General Properties)

A Ras proportional chamber consists of an anode wire surrounded by some gas and cathode planes. The passage of a charged particle through the chamber crates a track of electron-1on pass. The electrons drift :oward the anode wire. If the field strength around the anode wire is sufficlently large an avalanche develops, resulting in an amplification of the original ejectron pulst by as fuch as a factor of $10^{\prime}$.

The quantitles that can be measured are the time and pulse helght of the anode pulses, as well as the disiribution of the induced cathode pulses. Position informition 18 commoniy obtained for such a chamber by one or mote of several methods. The flrst and crudest wethod is that used in a smple multiwite-proportional cheabre (MhPC).l Such a chamber consists of a plane of anode wires. For particle trajectories at normal incidence pulse formation only occurs on a single anode wire. Determining shlch anode wire elred on the passage of a charged particle through she chamber determines 1 ta pustition with an accuracy glven by the anode wire neparation (the minimum practical separation 1s about Ima). A second method invelves 
determining the particle coordinate slong the anode wire. Two methods to accomplish this are to use the induced cathode pulses on a delay 112 and neasure the cathode distribution directly ${ }^{3}$ or to use a res. tive anode wire and use $a R^{4}$ or charge division type of readout. ${ }^{5}$ The chird method is to weasure the time it takes for an electron track to drift to the anode wire. 6 Such a measurement provides the shortest distance from the particle track to the anode wire, thus improving on the postion resolution obtainable for a given wire spacing over a simple MWPC. Several methods exist for determining on which side of the anode wire the track occurred.', 8

The ultimate position resolution obtalnable from such a detector is determined by the dynamics of the lon track formation and by the subsequent diffusion of the electrons in the time between the formation of the fon track and 1 ts detection. Since $1 \mathrm{n} 1 \mathrm{~cm}$ of Argon at atmospheric pressure (the typical thickness of a chamber) one out of 20 minimum londzing particles will eject an electron whose range is greater than $100 \mathrm{um}$, one can expect the ultimate position resolution from a gas proportional counter to be $\$ 100 \mathrm{um} / \sqrt{20}$ or approximately 20 wa $(a)$. Another contribution to the resolution is diffusion. In the direction parallel to the electric fleld, diffusion of the electrons in a drift chamber of lengths of $1 \mathrm{~cm}$ and with an Ar-Isobutane gas mixture contributes 40 wo to chamber resolucion. 9 it is interesing to nate that the diffusion in the direction perpendtcuiar to the electric field is typically 2 to 5 times soaller ${ }^{10}$ than in the direction parallel to the electric tield, and so the expected resolution is better in chambers which measure position along the directicn of the anode wire.

Although induced cathode pulse readouts are expected to give better resolutions than drift chambers, such resolutions have oni: been recently observed, and then only for small chamber areas. In f1R. I we show results obtalned by Charpak, et al. ${ }^{3}$ uaing a arall detector. The position for each event was found by independently reading out and digltizing the induced pulse heighes on aeveral cachode strips, and then computing tlie centrold of the induced charge. The beat resolution obtalned 10 70 um FWhM, abour a fector of 2 better than can be obtalned with a drift chamber. Sim!lar reoults 
have been obtalned using a delay-line readout chamber with ar. active area of $5 \times 5 \mathrm{~cm}^{2}, \mathrm{f} 1 \mathrm{~g} .2 .11$

The counting rate of a MWPC is limited by space charge effects. This limits the rate in a typical chamber to $\approx 105 / \mathrm{sec} / \mathrm{cm}$ of anode wire.9 In spectrometer applications, where events tend to be Localized on a chamber, such a rate can be exceeded at reasonably low singles rates. Another consideration which is sometimes important is the thickness of a chamber and consequent multiple scattering and energy straggling of the incident particles.

\section{Spectrometer Systems}

Although as mentioned in the previous section, one can obtain the best position resolution from MhPC's by finding position along the anode wire (either directly, using delay line, or with resistive anode wires), the best resolution of (lo0um has been obtained only recently. In chambers of reasonable length, $260 \mathrm{~cm}$, position resolution has been dominated by contribution from the methods used to read out the cathode information. The accuracy avallable from charge division'2 or cielay-l1 le readouts $3,14,15$ has been 1101 ted to "500 wm (FhT:). Because of the complexity of the readout systems, chambers which direcily digitize the induced charges on cathoda pickup strips, - method which is not expicted to degrade in long chambers, have not been implemented for large ipectrumeter systems. In fact, chambers in the focal flanes of the highest resolution medium energy spectrometers (HRS, Bates, EPICS) have used drift time information to interpolate hetween anode wires and consequently provide posilion information with reso:ution $150 \mathrm{um}$ (FWHM).

\section{A. Delay-line Readout Drift Chambers (DRDC)}

The chambers used to readout the HRS and EPICS unectrometer focal planed, more thoroughly dearrlbed elsewhere, 16 are delay-11ne readout drift chambers. These systems use chambers with dimensions of up to $30 \times 90 \mathrm{~cm}^{2}$. Four palro of chamber planes, two rrithogonal sets of four planes each, are used for each trajectory weasurement. The planes are of an alternating gradient designto with 8 mo wire opacings. Alternate fleld defining cathode wires are buesed together, providing two cethode outputs per plane, and the anode wires are coupled 
$-5-$

directly into a fast $(2.5 \mathrm{~ns} / \mathrm{cm})$ delay $11 \mathrm{ne}$ 15 whlch provides two anode outputs per plane. The anode planes, which are run ai high positive voltage, are separated by grounded foll cathode planes.

An assembly vlew of such a chamber is presented in fig. 3. The oring seals between each plane provide a gas seal that is suffictently tight to allow the chamber assembiy to $b$ : used in a vacuum. All connections to the chamber are made through the front cover plate using either BNC connectors for the cathode signals or SHW connectors for the anode signals. Entrance and exit windows are constructed of mylar. This chamber assembly, consist:ing of elght wire planes used in the front of the EPICS spectrometer and eperated 1: a vacuum with 51 mylar entrance and $\mathrm{cx}$ tit windows, presents $30 \mathrm{mg} / \mathrm{cm}^{2}$ of mass to the incident beam.

The anode signals are capacitively blocked and terminated into $100 \Omega$ at the input to the Lecroy systems Corp. LD, 04 amplifler-discriminator located approximately I weter from the chamber. The resultant NiM levil fast logic pulses are transported to the counting house through up to $200 \mathrm{~m}$ of $50 \Omega$ coaxial cable, and are then regenerated into a NIM leading edge discriminator and used as stops for CAMAC multichannel TDC's. The start signals for the IDC's are obtalned from the trigger colncidence logis of the spectromeier system, which can include information from the chambers. joarse position intumation is provided by the time difference between oulses from the crids of the delay lines whereas finer information ts obtalned from the ilme sum (drift time information)

The typical postion resolution obtained fror these chambers is $125 \mathrm{um}$ (FhrM) and 1s independent of the chasher size. This resolution is not compromised by counting rates of up to $10^{6} / \mathrm{sec}$.

B. Vertical Drift Chamber

A novel MhPC drift chaober called the vertlcal drift chamber (VDC) has been described by Ber:ozz1, et al..1' and used to read out the MIT energy Josn opectrometer at Bntes. Thie device uses a angle wire plane, rotaced $45^{\circ}$ with respect to the centrai ray leaving the spectrometer and dies apprnximately in the focal surface of the apectroneter. A detalled crose section view of inle chamber ts shown in 1 18. 4. Each of the actlue anode wires are alternately coupled 
through an amplifier and discriminator into one of three delay lines. Th1s readout scheme is shown in $\mathrm{fig}$. 5. As in the chamber described above, the difference in pulse arrival times from the ends of the delay lines is used to decode the wire number, whlle the sum is used to frovide the drift time.

As can easily be seen, with these chambers one plane provides both position and angle information. The accuracy of the reconstructed track position is $120 \mathrm{Hm}$ (FWFM) and the measured angle resolution is $12 \pi r$.

IV. Recent Developments

A. Use of Induced Cathode Pulses

An alternative method of making the Left-Righc decision recently applied to individual wire readout chambers 1,8 is to use the current pulses induced on the cathode fleld defining wires. Using the present $D R D C$, Left-Right information was obtalned by measuring the difference in the indused currents on the cathode wires on elther side of the active anode wire. To minimize the electronics needed for these measurements, every other cathode wire in a plane is bussed together. providing two cathode outputs per plane. The difference of these two signals is gcnerated using voltage-sensitive differential amplifier. For odd (even) wires, a positive (negative) signal is observed for particles passing to one side of the anode wire, and a negative (positive) signa! is observed for particles passing to the other side. The integration time constant of the amplifier, determined by the 80 pf apacitance of the chamber and a 5008 terminating resistor, is about $40 \mathrm{~ns}$. The analog ouput from such a circuit, Integrated for $100 \mathrm{~ns}$, 1s shown in $11 \mathrm{~g} .6$ as a function of position. One can see a clear separation between the $L-R$ algnals. Inttal tests of this methods showed an erroneous left-right decision was made approximately lt of the time.

Th1s method reduces the number of chamber planes required to make - position measurement to one, and consequentiy results in. consiferable aimplification of the spectrometer syatem. 
B. Reduction of Multiple Siatzering

The present $11 \mathrm{mit}$ of the resolution of the EPICS spectrometer is determined by the accuracy of the angle measurements made in the front chambers (a schematic view of the spectrometer system is shown in f1g. 7). The measured Fhrm of this angle determination 154 mrad, about what is expected from multiple scattering in the chamber gas. A reduction of a factor of $=2$ can be made in this multiple scattering by using a chamber gas mixture based on Neon racher than Argon. Initial tests of such a mixture showed it to be feasible, although some trouble was encountered with obtaining an efficiency greater than $98 \% / c h a m b e r$ plane. We hope to solve this problem with more experimentation.

A further reduction in the thickness and muliple scattering in these chambers can be obtained by replacing the existing windows with preformed Beryll1um windows. Windows of 0.001 " thickness can be made by sputering Beryllium onto an aluminum mandreil. Forming the windows to a spherical shape reduces the stress, and allows thinner windows to be used over a given area.

V. Conclusion

Most present spectrometer readout systems use drift chambers to provide the needed positive resolution. With such chambers, it is possible to measure positions with accuracies of $\approx 120 \mathrm{ww}$, and angles with accuracies limited by multiple scattering. The most cost effective spectrometer designs art likely to be ones which include the possibility of software corrections which can be made with such readout systems in the design phase. 
1. G. Charpak, R. Bouslier, T. Bessari, J. Favier and C. Zupansic, Nucl. Instr. and Meth. 62, (1968) 262 .

2. R. Grove, I. Ko, B. Leskovar and V. Perez-Mendez, Nucl. Instr. and Mpth. 99 (1970) 381.

3. G. Charpak, G. Petersen, A. Policarpo and F. Sauli, Nucl. Instr. and Meth. 148 (1978) 471 .

4. C. J. Borkowski and M. K. Kopp, Rev. Sci. Instr. 39 (1968) 1515.

5. B. G. Harvey, J. Mahoney, F. C. Pühlhofer, F. S. Goulding, D. A. Landis, J. C. Faivre, D. G. Kovar, M. S. Zisman, J. R. Miriwether, S. W. Cosper and D. L. Hendrie, Nucl. Instr. and Meth. 104 (1972) 21 .

6. A. H. Walenta, J. Heintz and B. Schürlein, Nucl. Instr. and Meth. $92(1971) 373$.

7. A. H. Lalenta, Nucl. Instr. and Meth. 151 (1978) 461.

8. A. Breskin, G. Charpak and F. Saul1, Nucl. Instr. and Meth. 151 (1978) 473 .

9. F. Saul1, CERN Report 177-09 (1977).

10. A. H. Walenta, Nucl. Instr. and Meth. 111 (1973) 467.

11. A. C. Thompson, J. M. Jaklev1C, B. H. O'Connor, C. L. Morris, submitted to Nucl. Instr. and Meth.

12. V. Radeka and P. Rehak, IEEE Trans. Nucl. Sc1., NS-25 (1978) 46. 
13. D. M. Lee, S. E. Sobottka, and H. A. Thiessen, Nuc1. Instr. arid Meth. 109 (1973) 421 .

14. J. L. Lacy and R. S. Lindsey, Nucl. Instr. and Meth. 119 '1974) 483.

15. C. L. Morris, H. A. Thiessen, and G. W. Hoffmann, IEEE Trans. Nucl. Sci. NS-25 (1978) 141.

16. L. G. Atencio, J. F. Amann, R. L. Boudrie and C. L. Mortis, Nucl. Instr. and Meth. 187 (1981) 381.

11. h. Bertozzi, M. V. Hynes, C. P. Sargent, C. Creswe11, P. C. Dunn, A. Hirsch, M. Leitch, B. Norman, F. N. Rad, and T. Sasanuma, Nucl. Instr. and Meth. 141 (1977) 457. 


\section{FIGURE CAPTIONS}

Fig. 1: Position resolution obtained by Charpak et al. ${ }^{3}$ for $X$-rays in a chamier with independently readout cathode strips.

F1g. 2: Position resolution obtained by Thompson et al.1l for X-rays in a short delay-line readout chamber.

Fig. 3: Assembly view of an EPICS front chamber. 16

Fig. 4: Schematic drawing of a vDCl/.

Fig. 5: Schematic drawing of the readout scheme for a VDC'?

Fig. 6: A scatter plot showing the difference in the induced cathode pulses for events on different sides of the anode wires. Anode wires are located every $8 \mathrm{~mm}$ from the certer of the plot.

Fig. 7: Plan view of the EPICS spectrometer as modified for a recent double charge exchange experiment. Chambers are located in both the front chamber box, as well as at the rear of the spectrometer. 
$-11-$

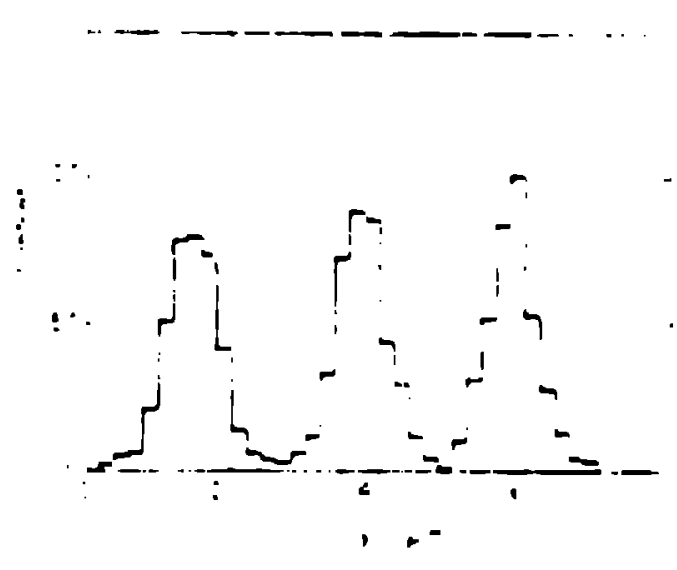

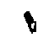

Figure 1 


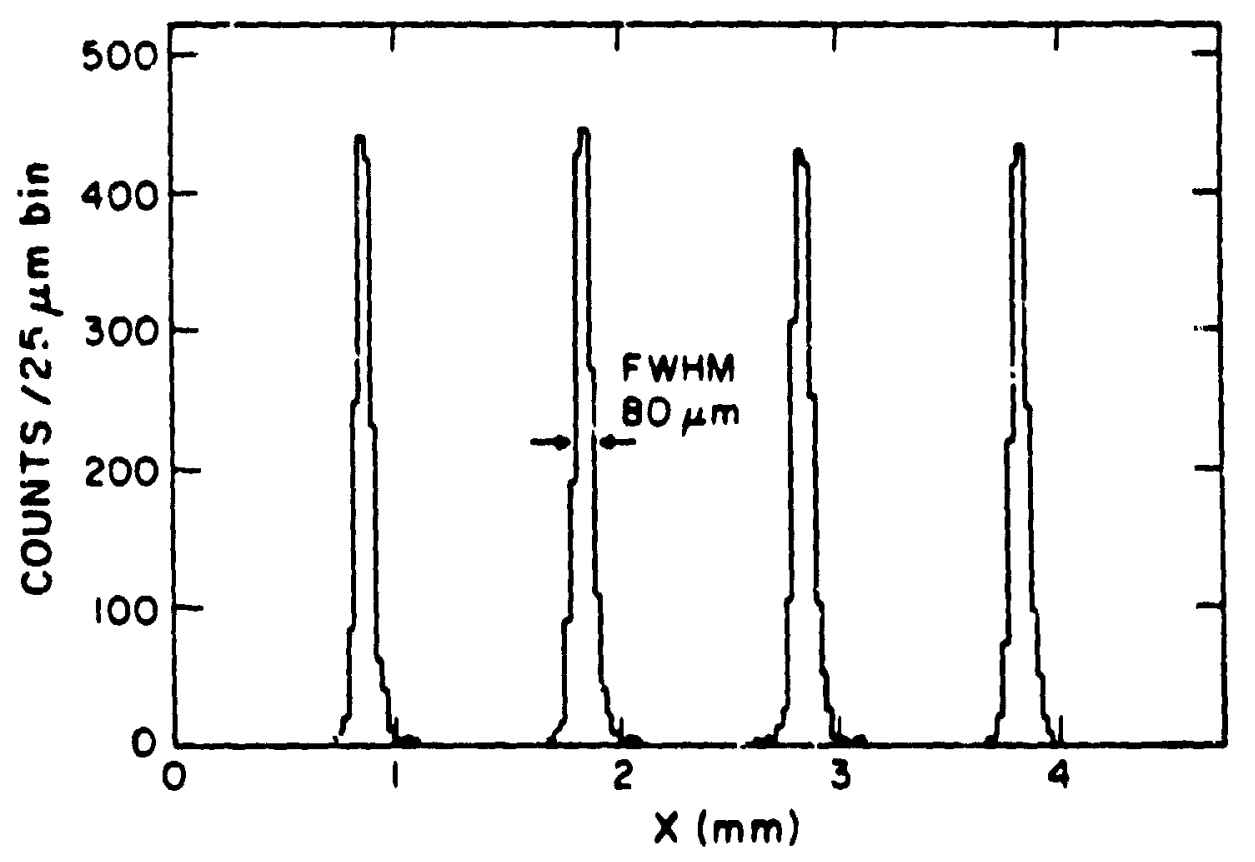

Figure 2 


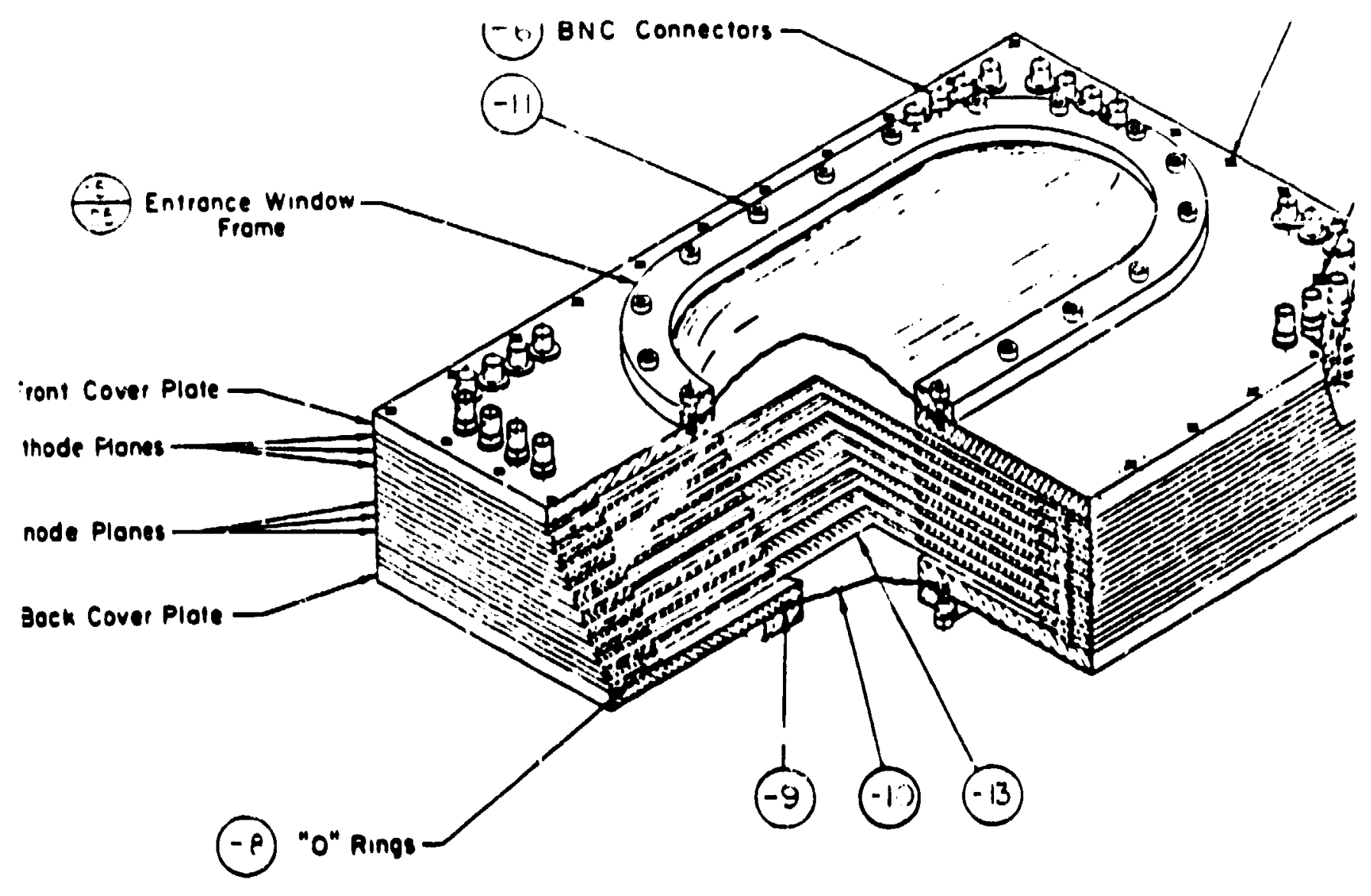

Figure 3 


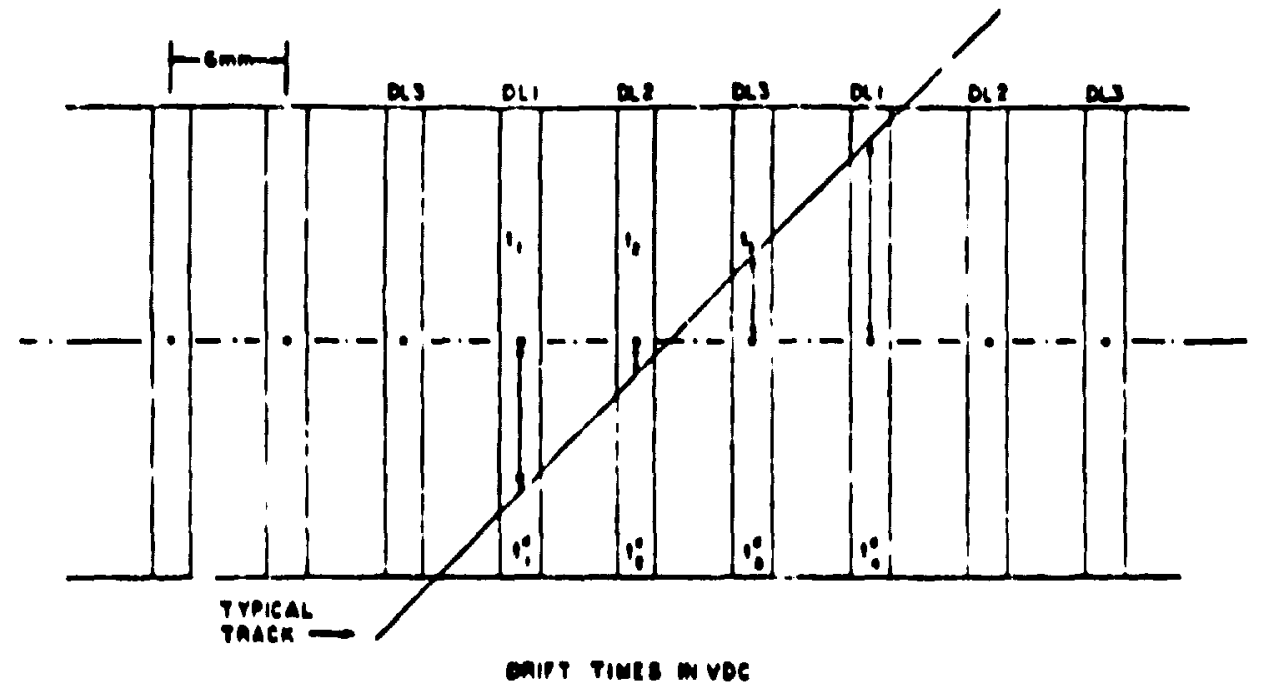

Figure 4 


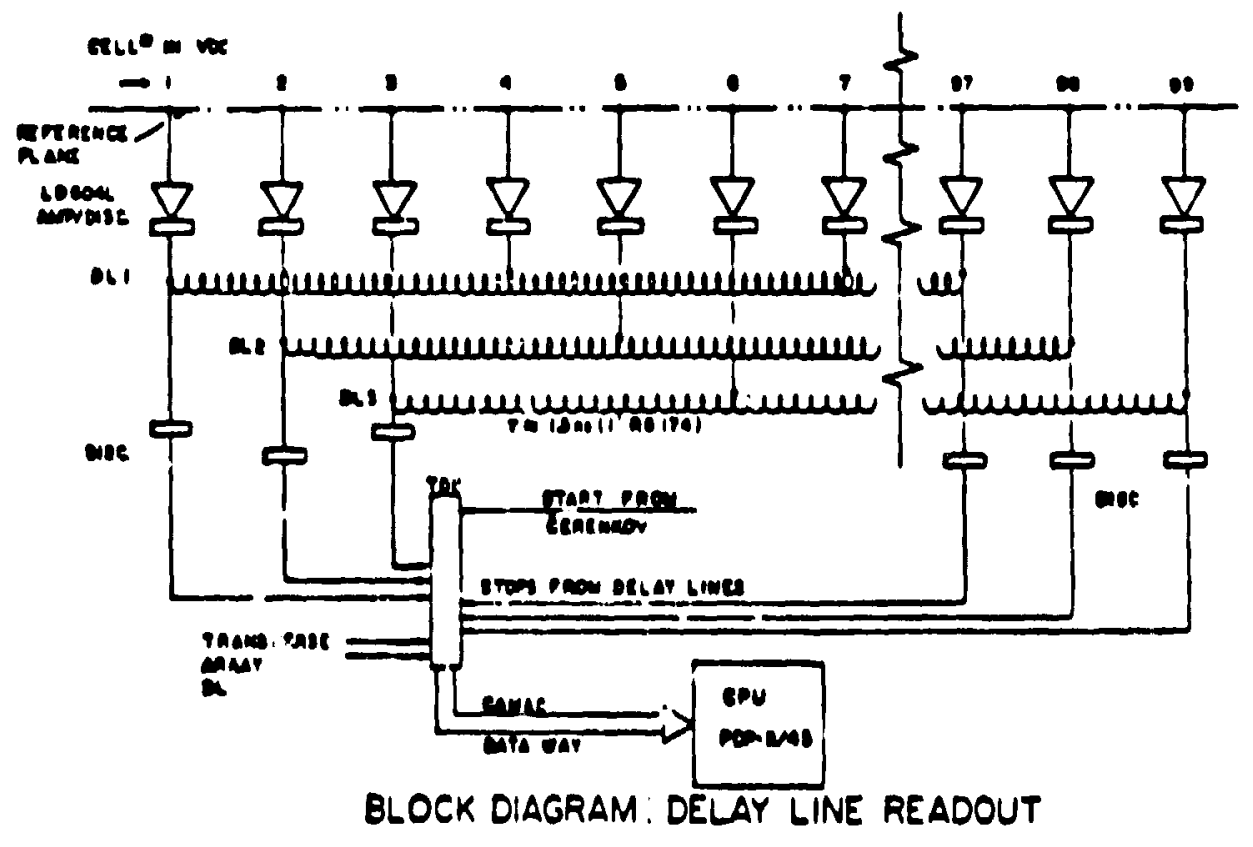

Figure 5 


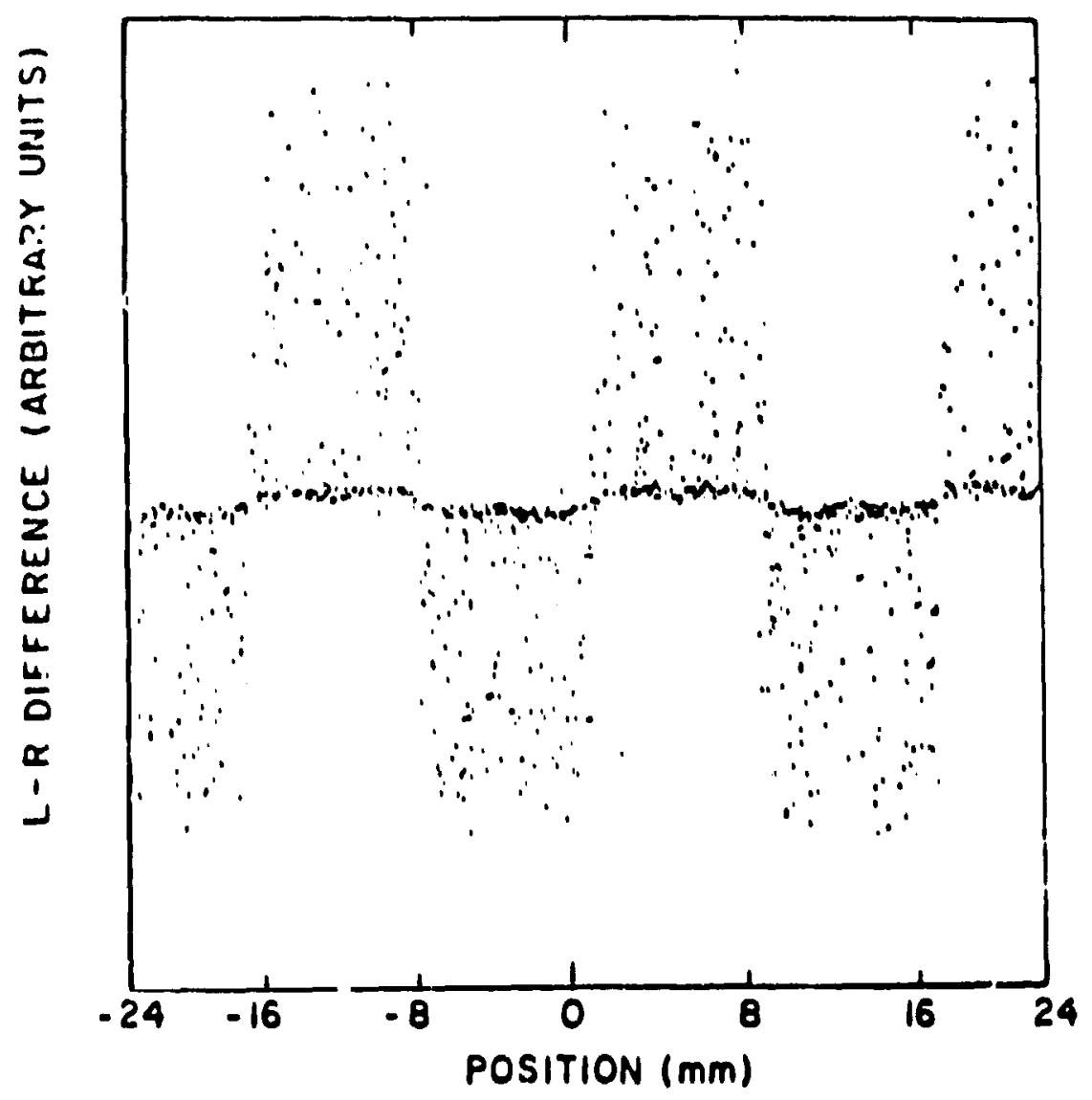

Figure 6 


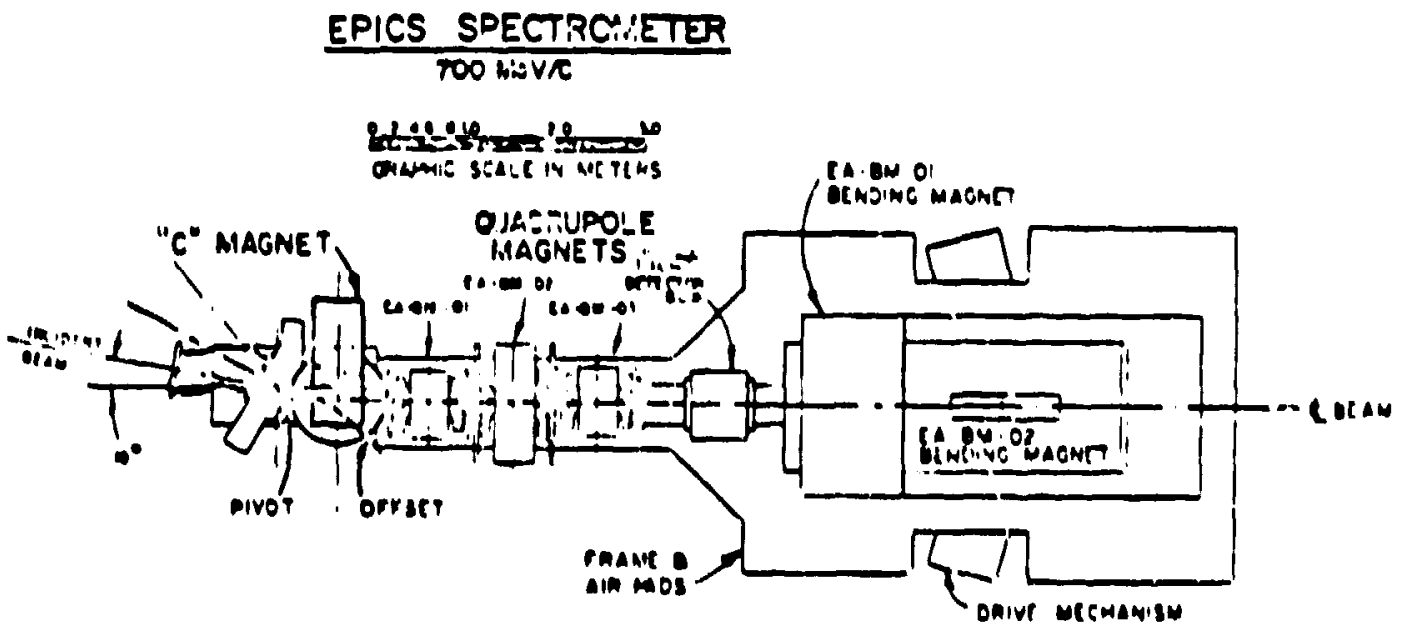

Figure? 